\title{
La escritura de un buen artículo científico en Educación y el entrenamiento de profesores universitarios en el discurso académico
}

\author{
Adriana Bolívar \\ Abolivar_2000@yahoo.com \\ https://orcid.org/0000-0002-1981-7796 \\ Universidad Central de Venezuela \\ Caracas, Venezuela.
}

Recibido: 28/04/2020 Aceptado: 19/05/2020

\begin{abstract}
Resumen
Desde que se empezó a difundir ampliamente la investigación sobre el discurso académico y profesional en el mundo de habla hispana (Parodi 2010; Bolívar y Parodi 2015), han ido en aumento los cursos sobre escritura académica para estudiantes universitarios en América Latina. No obstante, los profesores en el campo de la educación encuentran dificultades para escribir y publicar artículos científicos en revistas indizadas. El objetivo de este artículo es mostrar la complejidad de un proceso que abarca en la teoría varios planos: a) la definición de artículo científico, b) los tipos de textos que aceptan las revistas como productos de la investigación científica, c) las tradiciones discursivas en educación, y d) las estrategias de los investigadores para cumplir con el requisito de escribir un buen artículo científico. Nos remitimos a la experiencia llevada a cabo con profesores de Universidades y Escuelas Normales mexicanas en cursos y talleres sobre la escritura del artículo científico en educación. Para explicar el problema usamos como referencia teórica el análisis interaccional del discurso (AID) (Bolívar 2005a, 2007) y damos especial atención a los conceptos de género discursivo, estructura textual y estrategias discursivas. Los ejemplos se toman de un corpus de artículos de revistas de educación usados en los talleres. Se señalan aspectos discursivos y lingüísticos y las opciones que tienen los docentes para publicar artículos de investigación rigurosos y bien escritos. Se llama la atención sobre la necesidad de fomentar en los talleres la escritura de artículos científicos con investigaciones terminadas y de promover la escritura de otros textos que cubren las distintas etapas de la investigación.
\end{abstract}

Palabras clave: Artículo Científico. Educación. Talleres. Análisis del Discurso. Discurso Académico. Escritura Académica.

\section{A escritura de um bom artigo cientifico na Educaçao e o entreinamento em discurso acadêmico de professores universitários}

\section{Resumo}

Desde que se iniciou a difundir amplamente a pesquisa sobre o discurso acadêmico-profissional no mundo de fala hispánica (Parodi 2010; Bolívar y Parodi 2015), hão aumentado os cursos sobre escrita acadêmica voltada para estudantes universitários na América Latina. Não obstante, no campo da educação, profesores/pesquisadores encontram dificuldades para escrever e publicar artígos científicos em revistas indexadas. O objetivo deste artigo é mostrar a complexidade de um processo que abarca, na teoría, vários planos: a) a definição de artígo científico; b) os tipos de textos que as revistas aceitam como produtos de investigação científica; 
c) as tradições discursivas na educação e d) as estratégias dos pesquisadores para cumprir com o requisito de escrever um bom artigo científico. Balizamo-nos, aquí, na experiência levada a cabo junto a profesores de universidades e Escolas Normais mexicanas em cursos e oficinas sobre a escritura de arttigo científico na educación. Para explicar o problema, utilizamos como referencial teórico a análise interacional do discurso (AID) (Bolívar 2005a, 2007) e dedicamos especial atenção aos conceitos de gênero discursivo, estrutura textual e estratégias discursivas. Os exemplos são tomados de un corpus de artigos de revistas de educação, utilizados nas oficinas. Problemas linguísticos e discursivos são levantados, além de opções que têm os docentes para publicar artigos de investigação, rigorosos e bem elaborados. Apontamos, ao final, a necessidade de se fomentar, nas oficinas, a produção de artigos científicos resultantes de pesquisas concluídas e de se promover a escritura de outros textos que cobren as distintas etapas da investigação.

Palavras-chaves: Artigo Científico. Educação. Oficinas. Análise de Discurso. Discurso Acadêmico. Escritura Acadêmica.

\title{
Writing a good scientific article in Education and training university teachers in academic discourse
}

\begin{abstract}
Since the research on academic and professional discourse began to spread widely in the Hispanic world (Parodi 2010; Bolívar y Parodi 2015), courses for university students aimed at promoting academic writing in various disciplines have continued to grow. However, one of the problems that teachers/researchers in education point out is the difficulty they have to write scientific articles for indexed Journals. In this paper I focus on the experience carried out with teachers in Mexican universities and Escuela Normales in courses and workshops on the writing of a scientific article in Education. The objective is to show the complexity of a process that touches upon several dimensions: a) how a scientific article is conceived, b) the types of texts that are are accepted by Education Journals as products of scientific research, c) discursive traditions in Education, d) the strategies researchers use to comply with the requirements for writing a scientific article. We focus on the experience with teachers from universities and Escuelas Normales in México in courses and workshops on the writing of a scientific article in Education. In order to explain the problem, Interactional discourse analysis (IDA) (Bolívar 2005a, 2007) is used as a theoretical framework with attention to the concepts of discourse genre, text structure and discourse strategy. The examples are taken from a corpus of Journals in education. Linguistic and discursive problems are higlighted and the options teachers have for producing rigorous and well written research articles are given. We conclude with the need to promote the writing of articles on finished research projects and with writing other texts that cover the various stages of a research project.
\end{abstract}

Keywords: Scientific Article. Education. Workshops. Discourse Analysis. Academic Discourse. Academic writing.

\section{Introducción}

La escritura de un artículo científico es la última etapa de un proceso de investigación que puede haber durado meses o años, pero el artículo necesita ser publicado lo antes posible para que la investigación sea validada por una comunidad científica y los autores sean 
reconocidos y legitimados como investigadores. El acto de publicar se convierte entonces en una necesidad imperiosa para las universidades y para los investigadores, quienes no siempre reciben apoyo sobre la escritura de textos especializados. De ahí que los cursos y talleres para investigadores de escritura académica sean de gran ayuda y, cada vez más, las autoridades toman conciencia de que sus profesores necesitan adquirir esta experticia no solo a través de la práctica con sus pares sino con profesores de lengua, lingüistas o educadores que se han especializado en esta tarea. Los especialistas, por su parte, se enfrentan a un trabajo difícil porque su experticia debe abarcar la escritura en general (para enseñar a escribir de manera coherente), la escritura especializada en una disciplina (conocer los textos académicos de cada área) y el discurso de la investigación (estar enterados de las formas de investigar en cada tradición científica). Por este motivo, basándome en investigaciones previas sobre el discurso escrito en las humanidades (Bolívar 1999, 2004), daré especial atención al proceso de escribir un artículo de investigación en el campo de la educación que, al igual que otras disciplinas, tiene sus propias tradiciones discursivas y formas de construir el conocimiento (Beke 2011; Bolívar 2005b; Beke y Bolívar 2009). Al mismo tiempo, me concentraré en la experiencia personal de talleres llevados a cabo con profesores universitarios del área de educación, cuya meta fue obtener bases y orientación para escribir artículos publicables.

El argumento central que subyace en los talleres es que la escritura del artículo científico en educación no se da en el vació sino en contextos culturales en los que la investigación es el producto de interacción entre personas que junto con construir conocimiento se construyen a sí mismas discursivamente como grupo y como personas en los textos que escriben (Bolívar 2004, 2005a; Bolívar, Cruz y López 2019; Carlino 2006; Hernández Ramírez 2018; Mostacero 2014). En este sentido, es muy relevante conocer las tradiciones discursivas en educación y sus formas de aproximarse al conocimiento a través de los textos publicados por los miembros de la comunidad científica de educadores. Por lo tanto, necesariamente, debemos dar atención a nociones básicas como: a) los géneros discursivos, entendidos como modos relativamente estables de actividad lingüística compartida y reconocida por los participantes (ver Parodi 2008); b) la estructura textual, porque los géneros se materializan en patrones textuales o secuencias textuales que tienen funciones semánticas (de contenido) y pragmáticas (acciones discursivas) (Scott \& Thompson 2001); y c) las estrategias discursivas, porque quien construye 
el texto adopta un punto de vista en su disciplina y lo manifiesta escogiendo las marcas lingüísticas de posicionamiento más convincentes (Bolívar, Beke y Shiro 2010).

El artículo está organizado en cuatro secciones que cubren aspectos teóricos y prácticos. Primero, damos atención a la definición de un "buen" artículo científico (AC) y las implicaciones para el entrenamiento de profesores en talleres sobre el discurso científico. Segundo, analizamos un corpus conformado por artículos proporcionados por un grupo de investigación en México, y otros disponibles en INTERNET que fueron localizados al hacer búsquedas relacionadas con los intereses de los participantes. Tercero, examinamos las estrategias de los autores para adaptarse a las exigencias de lo que se considera un buen artículo científico.

\section{La definición de un "buen” artículo científico en educación}

Un artículo científico (AC) es un tipo de texto que se diferencia de otros textos académicos en varios aspectos: a) es un texto público cuyo objetivo es presentar ante una comunidad científica los resultados de una investigación (UNESCO 1983); b) sigue en su escritura pautas exigidas por la comunidad científica de pertenencia que, generalmente, adopta el patrón Introducción, Método, Resultados, Discusión, Conclusión ( IMRD/C), pero puede haber variaciones en cuanto al contenido de cada sección, por ejemplo Discusión y Conclusión pueden ir juntas o separadas dependiendo de la disciplina (Bolívar \& Bolet 2011); c) la investigación debe ser empírica, vale decir, debe haber datos cuantitativos, cualitativos, o de ambos tipos, d) la investigación debe ser (idealmente) repetible o replicable, lo que exige que el método y los procedimientos deben estar expuestos de forma clara y explícita (Titscher, Meyer, Wodak y Vetter 2000). El artículo científico (AC) se diferencia de un ensayo científico (EC) en que este es más evaluativo debido a que su objetivo es argumentar o defender una visión personal, preferiblemente con referencia a datos propios o de otros que apoyen los argumentos. Como veremos más adelante, la diferencia entre un AC y un EC a veces se hace borrosa en el campo de la educación.

Existen muchos manuales en inglés sobre lo que significa escribir un artículo científico en distintas disciplinas y también se encuentran bastantes publicaciones en español (Castelló 2007; Cisneros y Olave Arias 2012; Cubo de Severino 2011; Day 2005; Sánchez Upegui 2011, entre otros), pero son pocos los que se concentran exclusivamente en el campo de la educación, 
y menos los que dan sugerencias explícitas basadas en la investigación sobre el discurso científico en educación. Por eso, el trabajo de Murillo, Martínez y Belavi (2017) constituye una excepción valiosa y útil para los profesores de educación que desean obtener orientación sobre cómo publicar en revistas científicas. Estos autores ofrecen indicaciones a las que llegaron por tres vías: reflexionando sobre su propia experiencia de muchos años en la investigación pedagógica, revisando y comparando sugerencias hechas en otras disciplinas, y con base en un estudio exploratorio en el que examinaron un corpus de 20 artículos publicados en revistas indizadas de alto impacto escritos en revistas españolas y de otros países de habla hispana.

Lo primero que Murillo et al. (2017) señalan es que el estándar sugerido para estructurar un artículo científico en otras disciplinas como la medicina (IMRD) resulta extraño para los investigadores en educación donde predomina la secuencia IMRC, y que se observan diferencias en la Introducción y la Discusión. De acuerdo con sus datos, la Introducción en Educación contiene el problema de investigación y la revisión de la literatura, "que sirve de Marco teórico" (p. 8), y se refiere a cómo se estudió el problema y qué se encontró. En más de la mitad de los textos analizados, Murillo et al observaron que la Presentación está separada del Marco teórico especialmente en los artículos que llamaron "internacionales" (de revistas no españolas), pero ellos lo interpretaron como una cuestión de preferencias. Los datos examinados también mostraron que en Educación la Discusión no parece tener el mismo significado que en otras ciencias porque tiende a coincidir con la Conclusión. No obstante, señalan que los artículos que incorporaron una sección de Discusión separada de la Conclusión tienden a ser los mejores.

Como resultado del estudio exploratorio, con base en la experiencia y en los datos, Murillo et al (2017, p.8) afirman que un buen artículo científico en Educación tendría las siguientes partes: Introducción (o Presentación), Marco teórico (o Revisión de la literatura o Estado de la cuestión), Método (o Procedimiento), Resultados, Discusión, Conclusiones, Referencias y que, en consecuencia, la estructura sería IRMRDyC o IMRyC dependiendo de si se hace diferencia entre las partes o no. Los autores dan recomendaciones basadas en sus datos sobre la longitud de cada sección, el estilo, las formas de organizar la escritura, la importancia del título, el problema de la autoría, los resúmenes, las palabras clave, y la escritura de cada una de las partes con ejemplos de los artículos estudiados. Terminan con una toma de posición en defensa de mejorar la investigación para mejorar la educación: 
Investigamos en educación para cambiar una realidad que nos rodea y que nos duele, investigamos para que se tomen mejores decisiones en la política educativa, en el centro docente o en el aula, investigamos para construir una mejor sociedad a través de una mejor educación (...) Qué investigamos, con quién y cómo...y cómo difundimos sus resultados son decisiones que reflejan nuestra visión de qué queremos cambiar y hacia dónde, y con ello nuestra visión de sociedad. (Murillo et al 2017, p. 30).

Las sugerencias de Murillo et al tienen muchos aspectos positivos, pero hay un punto sobre el cual es importante llamar la atención ya que se relaciona con las diferencias entre los géneros de investigación en educación. Murillo et al. (2017) equiparan el AC con artículo de investigación empírica (como tiene que ser) y, por eso, estos investigadores evitaron seleccionar "artículos como ensayos y trabajos de meta análisis que, por su tipología, cuentan con características que hacen más compleja su comparación” (p. 6). Lo interesante es que lo que para estos autores fue una decisión metodológica en realidad oculta un problema fundamental para la investigación en educación y tiene implicaciones para la formación de investigadores y buenos escritores Se trata del hecho de que en las revistas científicas en educación (y en otras disciplinas) se da cabida a diferentes tipos de textos, cada uno con propósitos diferentes (reseñas, ensayos, editoriales, obituarios, foros, etc.) y eso no es difícil de observar. Pero el problema radica en que en algunas revistas de educación parecen existir similitudes entre artículos denominados científicos y otros como los foros, que son de discusión o evaluación. Esto se explica en parte porque los educadores tienden a favorecer el estilo más ensayístico en sus textos, vale decir, les interesa dar a conocer sus posturas ante los problemas sociales y de la educación con el fin de cumplir con su misión de orientadores.

Esta tradición discursiva se ha hecho evidente en investigaciones con corpus de artículos de investigación en revistas de educación. Por ejemplo, Beke (2011) encontró más similitudes que diferencias entre artículos de investigación y artículos publicados en la sección Foro de la Revista de Pedagogía, venezolana. Esta afirmación encuentra asidero en otro estudio anterior en el que Beke \& Bolívar (2009) estudiaron un corpus de artículos de investigación de varias disciplinas (Filosofía, Linguiística, Educación y Psicología) para averiguar de qué manera los autores construyen los grados de certeza y compromiso en sus afirmaciones mediante el uso de la modalidad epistémica y deóntica. Ellas encontraron que en los artículos de educación se usaba la modalidad deóntica (la del deber ser) mucho más que en las otras disciplinas, y que existían 
más semejanzas de estilo con filosofía que con psicología o lingüística. Por consiguiente, la definición de un "buen” artículo de investigación científica en educación tendría que tomar en cuenta esta tradición discursiva y, además, para efectos de entrenar a los profesores, habría que señalar la diferencia discursiva entre artículos de investigación empírica y otros que pueden exhibir variedad de estilos porque corresponden a distintas etapas de la investigación.

\section{Las bases teóricas y metodológicas de los talleres de entrenamiento}

Lo talleres de entrenamiento a los que nos referimos en este trabajo fueron dictados en México entre 2017 y 2019 y estuvieron dirigidos a profesores en el campo de la educación en Escuelas Normales y en Universidades ${ }^{1}$. Por lo tanto, los participantes tenían una formación heterogénea como profesores de distintas disciplinas (matemática, lengua materna, lengua extranjera, historia, psicología, etc.) y, al mismo tiempo, niveles diferentes de experticia ya que asistían profesores jóvenes que nunca habían escrito un artículo y otros pertenecientes a Grupos de Investigación integrados por doctores o en el proceso de obtener una maestría o doctorado. En ambos casos, era necesario tener en mente que había que dar mucha atención a la experiencia previa de cada uno, así como a las relaciones interpersonales para no afectar la imagen personal y grupal de profesores autorizados en un campo del saber.

\section{Los supuestos teóricos}

Los talleres fueron dictados tomando como referencia el análisis interaccional del discurso (Bolívar 2005a, 2007) en el que el texto escrito se concibe como una interacción entre el que escribe y el que lee. Por consiguiente, nos interesaba que los profesores/investigadores tomaran conciencia del proceso de construcción y escritura del artículo como dos acciones que se complementan. Por una parte, debían ubicarse en un campo de conocimiento, reflexionar sobre la relevancia de su investigación, tener claro el método que estaban usando, pensar en los interlocutores óptimos (a quién iría dirigido) y seleccionar la(s) revista(s) más apropiada(s) para

\footnotetext{
${ }^{1}$ Agradezco muy sinceramente a la Escuela Normal Oficial de Irapuato (ENOI), la Escuela Normal Superior Oficial de Guanajuato, la Escuela Normal Superior "Prof. Moisés Saenz Garza” de Nuevo León, la Benemérita Centenaria Escuela Normal de Guanajuato, así como a la Universidad Pedagógica Nacional, la Universidad Autónoma de Tlaxcala, la Universidad Autónoma de Querétaro, la Universidad de Quintana Roo, la Universidad Autónoma de Nuevo León y a la Universidad de las Américas Puebla, por sus amables invitaciones que me permitieron aplicar el análisis del discurso a la formación de investigadores y escritores, y al mismo tiempo, crear entrañables lazos de amistad.
} 
enviar su artículo. Por otro lado, tenían que tomar conciencia de que escribir el artículo científico significaba construir un texto que debía cumplir con ciertos requisitos y que, al escribirlo, desplegarían simultáneamente varios roles, especialmente el de escritor que se comunica con un lector a quien le da señales sobre la organización y contenido del texto, el de investigador que reporta sus hallazgos y su posición sobre un problema dado, y el de una persona con una historia propia, individual, cultural e ideológica.

También era importante introducir dos conceptos clave en el análisis del texto escrito, a saber, el plano de la interacción, que es lineal (una palabra, frase, oración o segmento tras otro) y el plano de contenido que es jerárquico porque se trabaja con temas o tópicos principales y secundarios (unos dentro de otros). En otras palabras, era necesario visualizar la escritura del artículo como proceso y como producto. Esto quiere decir que era importante diferenciar entre el proceso de ir y venir, de avanzar y retroceder en la investigación y el proceso de estructurar linealmente un texto que, en el caso del artículo científico, sigue una secuencia aceptada por la comunidad científica (IMRDyC o IMRC).

Era necesario recalcar que, en términos de las acciones que se llevan a cabo cuando investigamos, se sigue un orden de acciones que incluyen por lo general: i) tener primero escritos los Resultados, ii) luego tener clara la explicación detallada del Método, iii) hacer los ajustes necesarios al Marco teórico, iv) revisar/reescribir la Introducción, el Resumen y el Título. Pero había que tener muy claro que cuando se escribe el texto completo, la actividad es al revés, ya que los lectores verían las partes del artículo muy bien relacionadas desde el inicio: i) Título, ii) Resumen, iii) Introducción (Problema, objetivos, marco teórico, posicionamiento), iv) Planteamiento del problema o contextualización del problema, v) Método, vi) Resultados, vi) Discusión, vii) Conclusiones. A menudo sucede que las dificultades en la escritura del artículo o de trabajos de grado de maestría y tesis de doctorado surgen por no tener clara la diferencia entre proceso y producto, lo que lleva a que la comunicación del resultado de las investigaciones se convierta en un gran reto, no libre de frustración. De ahí que la investigación sobre la escritura de investigadores en formación es muy relevante, especialmente si se toman en cuenta las macro etapas de la investigación (Gonzalez 2014).

En vista de que, en algunos grupos, los asistentes a los talleres no tenían resultados de su investigación, reportaban resultados parciales, o no habían sistematizado los datos de acuerdo con las preguntas de la investigación, surgían problemas que afectaban la escritura del artículo 
científico porque se hacía difícil seguir la estructura del AC. También se daba el caso de investigadores a quienes les habían rechazado algún artículo o estaban en proceso de enviar su artículo a una revista y necesitaban revisarlo. El problema mayor era que, aun sin tener la investigación terminada, había profesores que se encontraban ante la imperiosa necesidad de publicar bajo la presión de los requerimientos institucionales. Para resolver este problema teníamos que referirnos a otras posibilidades de escritura, a otros tipos de textos científicos, y la tarea se hacía mucho más compleja debido a la amplia variedad de textos en este campo.

Por ejemplo, Sánchez Upegui (2011), después de examinar formas de comunicación científica en revistas especializadas, hace una división entre tipos textuales investigativos y académicos. Dentro de los textos investigativos incluye, a su vez, dos sub-tipos: el artículo científico, como resultado de proyectos de investigación, y otros textos que son resultados y/o avances de la investigación, entre los cuales se encuentran: artículos de reflexión teórica, de revisión, metodológicos, informes experimentales, balances historiográficos, estudios de caso, ensayos de informes clínicos, sistematización de experiencias (p. 95). Mientras que en los textos de divulgación académica, él hace una lista de variados tipos que, aunque no directamente relacionados con la investigación empírica, constituyen géneros que conducen a generar preguntas y, por ende, a la reflexión sobre la investigación, tales como: artículos analíticos, interpretativos, literarios, expositivos, anales, entrevistas, traducciones, artículos en blogs, reportes de análisis y de proyectos, y otros (p. 95). El foco de los talleres, en todo caso, tenía que ser los textos investigativos porque los textos académicos estarían en teoría más relacionados con la divulgación del conocimiento.

En nuestro caso, para los talleres sobre la escritura del artículo científico, necesitábamos concentrarnos solamente en el artículo científico como resultado de una investigación, y también en la escritura de otros artículos relacionados con el proceso de investigación. De ahí que introdujimos la diferencia entre artículo científico (AC) que sigue fielmente la estructura canónica (IMRC o IMRDyC) y el artículo de investigación (AI) que podría no seguir esta estructura o alterarla. La instructora (en este caso la autora de este artículo) tenía que conocer las investigaciones de los participantes (lo que se hacía conversando con cada uno) para poder orientar la escritura hacia un artículo de investigación cuantitativa, cualitativa, o mixta, en vista de que, en particular, la sección del Método y de los Resultados en los artículos serían diferentes 
dependiendo del método empleado (ver Murillo et al. 2017). Igualmente, era necesario tener en mente que no todos los artículos que se encontrarían serían de investigaciones culminadas.

\section{La metodología}

Las sugerencias existentes sobre la escritura del artículo científico en educación fueron muy útiles y las usamos en los talleres como marco de referencia global, especialmente las que fueron hechas con base en la investigación de artículos de calidad (Murillo et al. 2017), pero era importante que los profesores se enfrentaran a otros tipos de texto y pudieran comparar la diferencia entre artículos sobre investigaciones terminadas y otros que solo mostraban aspectos del proceso de investigación. Los objetivos eran, primero, identificar artículos científicos (investigaciones terminadas) y segundo, evaluar otros artículos que tenían otros propósitos. Eventualmente, la idea era que los profesores detectaran aciertos y fallas y tomaran conciencia sobre su propio proceso de escritura. Al mismo tiempo, nos propusimos profundizar aspectos relacionados con la investigación de los temas que interesaban a los/las participantes y formas de investigar en educación, lo que generaba discusiones sobre procedimientos en la investigación cuantitativa y cualitativa, y sus efectos en la escritura de los artículos.

Varios de los artículos empleados en los talleres fueron usados, al mismo tiempo, como textos de lectura para la discusión y como objetos de análisis, con el doble propósito de profundizar en los temas de investigación y de evaluar la escritura de sus autores. De forma paralela, cada participante trabajaba en el desarrollo de su propia investigación con diferentes objetivos: definir el título adecuado, precisar la relación entre título, resumen e introducción, revisar el planteamiento del problema, precisar el método, redactar los procedimientos, presentar resultados, escribir conclusiones, revisar las referencias.

Cada taller incluía una serie de tareas previas a la escritura y durante la escritura. La primera tarea de reflexión, para ubicarse como investigadores y explicar el estado de su investigación, consistía en una serie de preguntas cómo las siguientes, cuya función era obtener material para redactar el título y un resumen provisional (con variación según el grupo):

1. ¿Cuál es mi campo de investigación? ¿Dónde me ubico?

2. ¿Cómo puedo resumir mi investigación? (redactar el título)

3. ¿Para qué investigo esto? (problema, objetivos o preguntas) 
4. ¿Cuáles son los conceptos centrales que manejo? (teoría, quiénes son mis autoridades)

5. ¿Cuáles son los métodos que necesito? (relación entre método, objetivos y teoría)

6. ¿Cómo voy a proceder? (descripción de procedimientos)

Esta tarea exigía que revisaran lo que ya habían escrito y reescribieran o rehicieran partes de sus trabajos. Era un ejercicio muy interesante porque ante la pregunta ¿que estás investigando? la tendencia en muchos casos era "yo trabajo investigación-acción” o "yo uso el método etnográfico", pero les costaba definir el problema y el porqué de la investigación. En general, había más preocupación por hablar del método que de las preguntas que los llevaban a escoger un método. Descubrimos más tarde que este problema derivaba en ocasiones de lineamientos institucionales que favorecían un tipo de investigación en particular (como el caso de investigación-acción).

Tomando en cuenta que los temas de interés que surgieron en los talleres fueron, entre otros, la educación inclusiva, la tecnología educativa, el plagio, la investigación acción, la escritura del artículo científico, para los efectos de este artículo, nos concentraremos en un pequeño corpus de artículos en los que pusimos a prueba los criterios que nos permiten caracterizar un artículo científico como "bueno". La lista de artículos, obtenidos en su mayoría de internet en búsquedas temáticas se encontraron en redalyc y scielo, y fueron muy variados en cuanto a su estructura y con respecto del cumplimiento de los requisitos señalados por Murillo et al (2017). También se incluyeron textos publicados por un grupo de investigación en México $^{2}$.

\section{El corpus de textos empleados en los talleres y su categorización}

Para categorizar los artículos, partimos del supuesto de que en educación encontraríamos textos ubicables en un continuum entre artículos científicos de investigación empírica (AC) y artículos de investigación (AI), más cerca del género ensayo, aunque enfocados en diferentes etapas de la investigación (antes, durante, después). A continuación, en el Cuadro 1, se presenta un grupo de los artículos empleados en los talleres categorizados por tema, artículo, tipos de texto $(\mathrm{AC}, \mathrm{AI})$ y país de la revista:

\footnotetext{
${ }^{2}$ Los textos de este tipo fueron obtenidos en comunicación personal con la Universidad de Guadalajara donde un Grupo Académico estaba investigando el plagio. Agradezco al Dr. Antonio Ponce Rojo, Jefe del Departamento de Estudios en Educación, por proporcionarme gentilmente dos de los textos publicados por ellos sobre algunos resultados de la investigación (Hernández Islas et al. 2015 y Hernández Islas 2016).
} 
Cuadro 1. Los artículos del corpus de AC y AI

\begin{tabular}{|l|l|c|}
\hline \multicolumn{1}{|c|}{ Tema } & \multicolumn{1}{|c|}{ Artículo (ver detalles en referencias) } & $\begin{array}{c}\text { Tipo de texto } \\
\text { y país }\end{array}$ \\
\hline $\begin{array}{l}\text { El plagio } \\
\text { (en pregrado) }\end{array}$ & $\begin{array}{l}\text { Hernández Islas, M., Ponce Rojo, A.; Ortiz Lefort, V. y Vergara } \\
\text { Fregoso, M. (2015). }\end{array}$ & $\begin{array}{c}\text { AC } \\
\text { México }\end{array}$ \\
\hline $\begin{array}{l}\text { Investigación-acción } \\
\text { (revisión métodos) }\end{array}$ & Fernández, M. B. y Johnson, D. (2015). https://scielo.conycit.cl & $\begin{array}{c}\text { AI } \\
\text { Chile }\end{array}$ \\
\hline $\begin{array}{l}\text { El plagio } \\
\text { (Actitudes de } \\
\text { científicos) }\end{array}$ & Hernández Islas, M. (2016). & AC \\
\hline $\begin{array}{l}\text { Tecnología educativa } \\
\text { (Historia) }\end{array}$ & $\begin{array}{l}\text { Torres Cañizales, P.C.; Cobo, B. y Kendry, J. (2017). } \\
\text { https://www.redalyc.org/articulo.oa?id= }\end{array}$ & AI \\
\hline $\begin{array}{l}\text { El artículo científico } \\
\text { (sugerencias) }\end{array}$ & $\begin{array}{l}\text { Murillo, F. J.; Martínez-Garrido, C. y Belvi, G. (2017). } \\
\text { https://doi.org/10.15366/reice2017.15.3.001 }\end{array}$ & $\begin{array}{c}\text { Aenezuela } \\
\text { España }\end{array}$ \\
\hline
\end{tabular}

\section{Fuente: Elaborado por la autora}

Cuando analizamos detalladamente cada uno de los artículos de este corpus se observó que no todos cumplían con los requisitos de un buen artículo científico. Por consiguiente, las actividades se concentraron en evaluar qué tipos de textos eran, si $\mathrm{AC}$ u otros. Puesto que en casi todos los talleres, los participantes leían al inicio el artículo de Murillo et al (2017), este fue el primero en ser examinado. Los demás artículos fueron presentados de acuerdo con su cercanía o no al artículo científico (AC) en tres momentos diferentes (con variaciones según el grupo, si de iniciación o expertos).

\section{Las actividades: tres momentos}

Primer momento: Criterios para identificar un AC y diferencias generales con el AI

El primer tema era obviamente los criterios para identicar un artículo científico (investigación empírica). Una primera observación de parte de los profesores fue que el artículo de Murillo et al. (2017), usado para obtener sugerencias sobre la escritura de un buen artículo científico, no cumplía cabalmente con los requisitos de $\mathrm{AC}$ en cuanto a su estructura. Efectivamente, se trataba de un artículo de investigación que, aunque incluía un reporte de investigación, su objetivo era presentar "sugerencias" como resultados de la investigación exploratoria y de otras tareas, tal como se indicaba en el título: Sugerencias para escribir un buen artículo científico en educación. El artículo era coherente con los objetivos y con el método 
empleado para llegar a las sugerencias, pero su estructura indicada en los subtítulos confirmaba el carácter predominante de AI, a saber:

Titulos de las secciones en Murillo et al. (2017)

Introducción

1. El artículo científico y su estructura

2. Unas palabras acerca del estilo

3. Organizar la escritura

4. La importancia del título

5. La autoría: ¿cuántos firman, quiénes y en qué orden?

6. El resumen y las palabras clave

7. Presentación y marco teórico

8. Objetivos

9. Método

10. Resultados

11. Discusión y conclusiones

12. Las referencias como criterio de calidad,

13. Acabado el artículo, ¿dónde lo publico?

Referencias

Como puede observarse, con excepción de las palabras Introducción y Referencias, que no llevan número, todas las demás secciones corresponden a las partes del artículo científico sobre el que se dan sugerencias apoyadas en datos. Por consiguiente, el artículo se catalogoba como científico por ser producto de la investigación científica (AI), pero su estructura no encajaba con la de artículo cientifico (AC) que, de acuerdo con la definición, presenta los resultados de una investigación empírica. Esto se puede comprobar lingüísticamente de varias manera: a) en el título mismo, b) en el resumen que tiene el objetivo de dar "sugerencias útiles" ("aportamos algunas sugerencias", "estas ideas surgen de tres fuentes", ... "a lo largo del artículo aparecen recomendaciones"... “aspectos a tener en cuenta por los autores"); c) en el lenguaje evaluativo empleado desde el inicio ("No hay atajos. Un buen artículo de investigación científica es el resultado de una buena investigación. Si no hay ésta, no puede haber aquel...” p. 6); y d) no hay sección Resultados, Discusión, Conclusiones.

\section{Segundo momento: El artículo científico y la variación}

El segundo paso consistió en examinar artículos científicos que cumplían fielmente con los requisitos de un AC y de averiguar qué diferencias había entre ellos. Como uno de los temas escogidos fue el plagio en las universidades mexicanas, revisamos artículos escritos por 
investigadores de una universidad mexicana, que resultó ser la Universidad de Guadalajara. Por lo general, en los talleres se analizaba primero un artículo escrito por varios autores (ACG: artículo científico de grupo) y se comparaba con otro escrito por una persona (ACI: artículo científico individual).

El primer artículo, escrito por un grupo (ACG) (Hernández Islas et al. 2015), constaba de 21 páginas, y mostró la siguiente estructura: 1. Introducción, 1.1. Revisión de la literatura, 1.2. Preguntas de la investigación, 2. Material y método, 2.1. Participantes y técnicas de recolección de información, 2.2. Método de análisis, 2.3. Validez y consistencia, 3. Resultados (12 páginas y media con sub-secciones), 4. Discusión y Conclusiones. Referencias. El análisis indicó que este artículo cumplía com todos los requisitos de un artículo científico (AC) y que incorporaba subtítulos muy precisos y detallados para las secciones y sub-secciones. No llevaba número en las Referencias. El segundo artículo, escrito por una sola persona (ACI) (Hernandez Islas 2015), tenía 15 páginas y, aunque perteneciente al mismo grupo de investigadores, presentaba diferencias de formato pues no había números em ninguna de las partes y se incorporaron secciones separadas, que en el artículo de grupo iban en la Introducción. Su estructura era: Introducción. Propósito de la investigación. Revisión de la literatura. El estudio realizado (Participantes, Abordaje metodológico). Resultados (4 páginas y media). Conclusiones. Referencias.

La comparación entre los artículos indicó dos cosas. El artículo de grupo cumplía fielmente con las sugerencias para un buen artículo científico (como lo indicaron los datos de Murillo et al. (2017) y el artículo de una persona mostraba diferencias de estilo que, aunque no le quitaban su carácter de científico, despertaba comentarios sobre lo innecesario de poner secciones diferentes para los Objetivos y la Revisión de la literatura y sobre el hecho de no destacar el método en una sección aparte ya que la autora lo había puesto dentro de la sección denominada "El estudio". Con excepción de estas observaciones, el artículo fue muy bien recibido por los participantes porque presentaba una muy buena revisión bibliográfica y los resultados generaron bastante discusión. Además nos sirvió para explicar aspectos lingüísticos clave como veremos más adelante en la sección 4.

Es importante agregar en este punto que una de las primeras señales de que estamos frente a un artículo científico empírico bien escrito se encuentra en el Título y en el Resumen 
porque ambos deberían ser coherentes con la estructura global del artículo. El Resumen sigue también el patrón IMRC. Veamos los resúmenes del ACG y del ACI para apreciar como sucede esto.

\section{Título y Resumen del ACG}

Estrategias docentes emergentes para prevenir el plagio en alumnos de pregrado. Un estudio fenomenográfico con profesores experimentados

En este artículo se presentan los resultados de un estudio de corte cualitativo realizado con profesores de pregrado en la segunda universidad publica más importante de México. El objetivo principal ha sido dar cuenta de las estrategias implementadas por los docentes ante el plagio académico, práctica no deseable que se está volviendo cada vez más frecuente en las aulas universitarias como uno de los efectos negativos de la creciente disponibilidad de grandes cúmulos de información en línea. El estudio, de corte cualitativo, se centro en aquellas prácticas que algunos profesores, con una antiguedad en la docencia mayor de 10 años implementan para "regresar" a la enseñanza tradicional bajo la cual fueron formados y con base en la cual también han realizado gran parte de sus labores docentes. La recolección de los datos fue realizada empleando entrevistas a profundidad y como método de análisis se empleó la fenomenografía. Los resultados permitieron establecer dos grandes categorias de prácticas emergentes, la primera tiene que ver com aquellas encaminadas a la prevención del plagio y la segunda, con las que se orientan a la detección y sanción del mismo. Sin embargo, también se pudo identificar como en la práctica, las estratégias implementadas no van realmente dirigidas al plagio, sino a conductas y hábitos que los professores asumen como relacionadas con este. El análisis da cuenta también de las posibles razones por las cuales se presentan estas prácticas de regreso, entre las que se tiene a los resultados de experiencias previas directas o indirectas y las dificultades para identificar nuevas formas de trabajo a partir de nuevos medios.

Palabras clave: Estrategias docentes, comportamiento no-ético, pregrado, internet (Hernandez Isla et al. 2015)

El resumen del ACG indica con claridad el tipo de estudio (de corte cualitativo), el objetivo, el problema (el plagio, efectos negativos, las prácticas de algunos profesores), el método (la recolección de los datos, entrevistas, la fenomenografía), los resultados, y las conclusiones, que están implícitas (posibles razones). Las palabras clave retoman palabras del resumen, con excepción de internet, pero alude a información en línea que aparece en el texto. Hay relación entre el contenido del título y del resumen. El artículo respeta este orden y proporciona la información anunciada. 


\section{Título y Resumen del ACI}

El plagio académico en la investigación científica. Consideraciones desde la óptica del investigador de alto nivel

El objetivo principal de esta investigación es contribuir a la comprensión de las formas en que los investigadores conciben el plagio académico y a quienes incurren en acciones de este tipo. Asimismo, busca comprender la manera en que los investigadores toman decisiones ante casos de plagio cuando ellos mismos están implicados como evaluadores o editores de publicaciones científicas. Para esta investigación, de corte cualitativo, se utilizaron entrevistas a profundidad para dar cuenta de la experiencia y percepciones narradas por 51 investigadores mexicanos de alto nível. Los resultados develan contradicciones entre la importancia que los investigadores dicen conceder al plagio y su percepción de cercanía del fenómeno, con las acciones emprendidas por ellos a propósito de éste y las experiencias vividas a lo largo de su trayectoria. Asímismo, permiten identificar algunos de los criterios que prevalecen para tomar la decisión de no ir a fondo en casos de plagio.

Palabras clave: ética profesional, investigadores, investigación científica, producción científica (Hernández Islas 2015)

En el resumen del ACI, cuyo texto modificó en parte la estructura, encontramos también los objetivos (el objetivo principal, asimismo), el método (cualitativo, entrevistas a profundidad), los resultados (contradicciones, criterios que prevalecen). Pero en este caso, los resultados y las conclusiones se confunden porque no hay referencias explícitas a las conclusiones. Las palabras clave se relacionan con el resumen, aunque ética profesional y producción científica no se mencionan en el texto. Hay relación clara entre el título y el resumen. El artículo completo sigue el orden anunciado, supera la información dada en el resumen y separa claramente los Resultados de las Conclusiones.

El tercer momento. Artículos de investigación variados (AI)

La revisión de artículos que no encajaban en el patrón recomendado para un AC generó mucha discusión y trajo a luz problemas de diferentes tipos, desde críticas a la rigurosidad de las revistas en cuanto a sus exigencias, las estrategias lingüísticas de los autores para que su texto se adaptara a la secuencia IMRC aunque no se tratara de una investigación empírica terminada, y la organización que pueden tener otros textos. Veamos los siguientes resúmenes y palabras clave de los artículos que catalogamos como AI de tipo ensayo de tres tipos: analíticoargumentativo, revisión de contenidos y revisión crítica de la literatura. 
Resumen 1: AI, analítico-argumentativo

De la educación especial a la educación inclusiva

La escuela de integración surge como una opción innovadora frente a la escuela anquilosada tradicional y excluyente; se define por su apertura y aceptación en las aulas regulares, a alumnos sin distinción de sexo, edad, origen social, situación económica, pertenencia social o étnica, así como a alumnos con necesidades especiales de aprendizaje que, tradicionalmente, eran excluidos o expulsados de la escuela regular. El objetivo del artículo es analizar, en una primera parte, la política educativa de los últimos sexenios en relación con la educación especial y su tránsitoretroceso hacia la escuela inclusiva y la manera como ha sido concebida la educación especial y la escuela inclusiva en nuestro país. Posteriormente, tratamos de demostrar el viraje de la política educativa hacia la vieja concepción de la educación especial, en detrimento de los grupos excluidos y más vulnerables socialmente, a pesar de los planteamientos de la necesidad de construir mayores márgenes de equidad, igualdad e inclusión económica, política, social y cultural, dentro de los cuales incluimos la educación por parte de Estado. Finalmente, en un tercer momento, concluimos con la propuesta renovada de una escuela inclusiva.

Palabras clave: educación inclusiva, integración, política educativa, normas de ingreso, discapacidad. (Juárez Núñez et al 2010)

Este resumen es diferente de los anteriores tipo AC ya que se nota inmediatamente que tiene otra finalidad, no en función de ofrecer resultados sino de evaluar una situación, lo que permite que lo categoricemos como ensayo de investigación (EI), diferente del ensayo académico (EA) que es más general (ver Castro, Sánchez y Hernández 2010). En este caso la secuencia textual es del tipo Situación-Problema (escuela de integración versus escuela anquilosada), objetivo (analizar la política educativa, especialmente la educación especial en dos sexenios en "nuestro pais"), Argumentación (demostrar el viraje hacia la vieja concepción), Propuesta (una renovada escuela inclusiva).

Un punto clave para categorizar el artículo como EI es el contexto en que fue publicado. En este ejemplo, se trata de una revista mexicana y los autores esperan que el lector entienda la situación y que sepa a qué se refieren los "sexenios" (períodos de gobierno que duran seis años en México). Por lo tanto, el tema, el problema planteado y el modo discursivo argumentativo están justificados ya que el artículo apareció en la modalidad dossier de la revista, vale decir, un número temático dedicado especialmente a "Procesos educativos en América latina: política, mercado y sociedad”. La meta era analizar los procesos educativos en México y, por eso, en el artículo no hay una sección de método ni resultados. No obstante, es interesante observar que dos de las palabras clave (normas de ingreso, discapacidad) no aparecen en el resumen aunque hay referencia indirecta a ellas (grupos excluídos, educación especial). El artículo completo 
sigue en general la estructura del Resumen. El estudio más detallado de este artículo trajo a la superficie la importancia de que los investigadores sean también buenos argumentadores y manejen bien el léxico referencial y evaluativo en los ensayos de investigación.

Resumen 2: AI, revisión de contenidos

Tecnología educativa y su papel en el logro de los fines de la educación

Este trabajo está dirigido a develar el papel que tiene la tecnología educativa en el logro de los fines de la educación. Para alcanzar esta empresa se aplicó la técnica de análisis de contenidos a diversas obras y publicaciones referidas a la temática del estudio, así como a documentos emitidos por organismos internacionales y nacionales con inherencia en materia educativa. $\underline{\mathrm{Se}}$ determinó que la educación puede lograr sus finalidades más trascendentales mediante el uso sistemático de la tecnología educativa, que emplea diversos medios y recursos para el aprendizaje escolar, ya sean los tradicionales (libros, pizarra, entre otros), o las herramientas que ofrecen las tecnologías de información y comunicación (TIC); concepto que no puede considerarse equivalente al de tecnología educativa.

Palabras clave: Educación, tecnología educativa, tecnologías de información y comunicación, Venezuela. (Torres Cañizales et al, 2017)

Este resumen anuncia un ensayo descriptivo equivalente a una revisión de la literatura, aunque está implícita una toma de posición. La estructura del resumen no está está indicada de manera directa ya que no se usan las palabras objetivo, método (aunque se dice técnica), resultados, conclusiones, pero sí se encuentran señales lingüísticas que permiten inferir la organización general: el tema (la tecnología educativa en educación), el objetivo (develar el papel..), el método (para alcanzar esta empresa, análisis de contenidos, diversas obras, publicaciones, documentos), resultados (se determinó que/ puede lograr...uso sistemático..TIC), conclusión (TIC no equivalente a tecnología educativa). Este texto da evidencia de lo consciente que están los investigadores de la obligatoriedad de seguir la secuencia recomendada (IMRC) aunque sea de manera indirecta en una revisión bibliográfica. La referencia a "análisis de contenidos" en el resumen es imprecisa ya que no hay una fuente que indique el tipo de análisis. Este problema se subsana en la Introducción del artículo donde está claro el problema y se indica la forma en que se analizaron los documentos para cumplir con el objetivo indicado, que fue ver la evolución de la tecnología educativa y los fines de la educación tal como están establecidos en la Constitución venezolana. La estructura del artículo confirma que se trata de un AI que se organiza temáticamente, como sigue: Introducción- Una mirada a la evolución de la tecnología 
educativa- Tecnología educativa en la actualidad-Fines de la educación- Fines de la educación y tecnología educativa- Conclusiones-Referencias bibliográficas.

El artículo está bien documentado y actualizado. Los fines de la educación se analizan y evalúan de acuerdo con organismo internacionales (ONU, UNESCO) y nacionales (Ley Orgánica de educación, Constitución de la República Bolivariana de Venezuela y otros), así como el papel de la tecnología educativa y de la tecnología de la información desde una perspectiva histórica. No obstante, este artículo llama la atención por su carácter más descriptivo que crítico, lo cual se hizo evidente en los títulos y sub-títulos que orientaban a los lectores hacia una relación de contenidos. En el fondo hay un posicionamiento que no se destaca en los sub-títulos porque sus autores favorecen la descripción sobre la toma de posición frente a un problema.

Resumen 3: AI, revisión crítica de la literatura

Investigacion-acción en formación de profesores: desarrollo histórico, supuestos epistemológicos y diversidad epistemológica

En el contexto de la formación docente, la investigación-acción ha tenido una importante influencia en las últimas tres décadas siendo usada frecuentemente como herramienta pedagógica. Nuestro objetivo fue analizar el desarrollo histórico de la investigación-acción educacional, conceptualizar sus aspectos comunes y profundizar algunos enfoques particulares. Desde un punto de vista teórico, la investigación-acción reconoce una historia común y una pluralidad metodológica. Metodológicamente realizamos una revisión de la literatura internacional y nacional. El resultado es el análisis y ejemplificación de tres enfoques particulares: autoestudio, indagación sobre la indagación e investigación docente. Concluimos que la investigación-acción educacional, en su unidad y pluralidad metodológica, ofrece diversas posibilidades a formadores de profesores y futuros docentes para reflexionar e indagar acerca del proceso de convertirse en profesor.

Palabras clave: investigación-acción; formación de profesores; autoestudio; indagación; investigación docente (Fernández \& Jonhson 2015)

Este resumen también corresponde a un ensayo de investigación cuyo propósito es revisar críticamente la literatura sobre investigación-acción. Los autores siguen la estructura IMRC de manera explícita aunque el artículo no puede catalogarse como AC. Se justifica el tema (la influencia de la investigación- acción como herramienta pedagógica), se da el objetivo (analizar ...conceptualizar...profundizar), la metodología (revisión de la literatura), el resultado (ejemplificación de tres enfoques), la conclusión (posibilidades para reflexionar e indagar...). 
El artículo es claramente un ensayo en el que la revisión de la literatura se convierte en una metodología que presenta primero los conceptos clave en la investigación - acción y culmina con la lectura detallada y crítica de tres enfoque escogidos. La estructura del artículo es la siguiente: Introducción- Desarrollo analítico (incluye siete sub-secciones con sub-títulos, cubre aspectos teóricos)- Método-Resultados- Discusión y Conclusiones- Notas-Referencias. El artículo llamó la atención por la meticulosidad de sus autores para explicar la revisión bibliográfica.

De hecho, los enfoques que escogieron fueron analizados con la misma técnica en el mismo orden: descripción del enfoque o movimiento académico, análisis de un estudio como ejemplo (contexto, objetivos, análisis realizado, resultados, fortalezas y debilidades). En las Conclusiones, los autores señalan:

Nuestro esfuerzo se ha enfocado en contribuir a la claridad conceptual (epistemológica) y la diversidad metodológica en la investigación-acción educacional en formación docente la cual tiene un fuerte potencial para contribuir al proceso de convertirse en profesor. Así, el objetivo de este artículo ha sido también pedagógico (Fernández \& Johnson 2015, p. 105).

En realidad, este es un ensayo riguroso que se plantea un problema epistemológico y obtiene resultados de una lectura profunda que sirve como metodología para revisar las opciones que ofrece un método ampliamente usado en educación. Los profesores asistentes al taller que investigaban este campo aprovecharon este artículo con respecto del contenido y como modo de organizar la revisión de literatura.

\section{Aspectos discursivos y linguísticos: las estrategias}

Como se podía esperar, muchas de las actividades en los talleres fueron dirigidas a la toma de conciencia crítica de los géneros científicos y de los recursos linguísticos empleados por los autores de los artículos publicados. Aunque no se hizo un estudio para medir los avances entre el antes y el después de los talleres, la experiencia indica que se tomó mayor conciencia sobre lo que significa enfrentarse al discurso cientítico y académico a partir del análisis de textos "reales" ya publicados y de la evaluacion de la escritura propia. Se puso en evidencia que la escritura de un artículo científico está relacionada directamente con la cantidad y la calidad de la investigación llevada a cabo individualmente y en grupo, así como con las prácticas sociales 
y discursivas relacionadas con el proceso de investigación (por ejemplo, la práctica social de trabajar solos, aunque formaran parte de un grupo académico y la práctica discursiva de no llevar siempre un registro escrito riguroso del proceso de la investigación, de los datos o de los procedimientos de la investigación). Igualmente, se tomó conciencia de que la escritura de un artículo lleva consigo el manejo de estrategias discursivas y lingüísticas que pueden garantizar una comunicación científica de calidad, porque con ellas se da evidencia de la coherencia global y local, del manejo del conocimiento proprio y ajeno y de que el autor o autora tiene conciencia de su persona como investigador(a). A continuación presentamos un breve recuento de algunos de los aspectos discursivos y lingüísticos que fue necesario trabajar de manera especial.

\section{La estructura y organización del texto: coherencia global y local}

Aunque los grupos estaban integrados por profesores que ya manejaban la escritura en general, fue necesario llamar la atención sobre las relaciones de coherencia global, especialmente a través de señales léxicas en los títulos y subtítulos de las partes del texto, que mantienen el tópico global. Igualmente, en el nivel local, fue necesario recordar el uso de conectores, especialmente para agregar información o argumentar. Por supuesto que la estructura IMRC fue una de las guías para evaluar tanto los resúmenes como los artículos haciendo la salvedad de que el AC y el AI eran géneros diferentes, pero también se conocieron patrones textuales como Situación-Problema- Evaluación y la estructura básica InicioSeguimiento-Cierre para constatar la cohesión textual y la coherencia del discurso (ver Bolívar 2005a). Aspectos estructucturales de los textos, relacionados con el uso de la gramática, la retórica y la cognición, han sido útiles para identificar diferencias entre escritores competentes y no competentes (Bustamante 2011).

\section{La construcción del conocimiento propio y ajeno}

Este aspecto fue tal vez el más estudiado debido a que había que enfrentarse a dos problemas. Por un lado, la (re)presentación del autor o autores en el texto en sus diferentes roles como autores, investigadores, argumentadores, evaluadores (Flotum 2009, p.112) y, por el otro, cómo diferenciar el conocimiento propio del ajeno. Se hicieron ejercicios y lecturas poniendo atención en el uso de los pronombres personales (yo, nosotros, tanto incluyente como excluyente, y al uso del se) y sobre las citas y referencias, no solamente para seguir normas (APA u otras) sino para comprender la función discursiva de las citas en el texto de modo que 
fuera evidente lo que el autor del texto sabía o pensaba y lo que atribuía a otros (Hernández Ramírez 2018). Aquí fueron necesarios y productivos los conceptos de citas integradas (con función gramatical en la oración) y no integradas (entre paréntesis en cualquier parte del párrafo) (Bolívar 2004, Beke 2011, Swales 1986, 1990). También se tomaron en cuenta otros aspectos como el uso de los verbos, para indicar diferentes procesos semánticos, y la diferencia entre léxico referencial y evaluativo. El uso de los verbos de reporte, en particular, resultó ser un problema debido a que, en ocasiones, el repertorio lingüístico era escaso o no había consciencia de los tipos de verbos y sus funciones en el texto investigativo (por ejemplo, diferenciar entre verbos cognitivos (creer, pensar, determinar), de investigación (averiguar, mostrar, demostrar, medir, recoger, etc.) y de argumentación (plantear, aceptar, rechazar, cuestionar, criticar), ya que no es lo mismo mostrar que demostrar o exponer que argumentar o determinar que concluir. De esta forma se creaba conciencia sobre un proceso de creación científica en el que la voz del autor y las voces de otros interactúan para producir el texto final mediante el manejo experto de recursos linguísticos clave para mostrar sus resultados, su subjetividad y su posición en un contexto cultural particular (Fløttum 2009, García Negroni 2008, Gee 2008; Mostacero 2014).

\section{La diatriba personal- impersonal}

Uno de los aspectos que había que trabajar de forma especial tenía que ver con la creencia casi establecida de que el artículo científico "debe ser impersonal”. Sobre este punto se hacían ejercicios para mostrar que el posicionamento del autor es ineludible porque en, (casi) cualquier interacción, la evaluación es inevitable (Bolívar 2005a, Bolívar, Beke y Shiro 2010). Igualmente, se mostraban resultados de investigación en los que se estudiaban ejemplos en los que el uso del llamado "se impersonal" no era tal en todos los contextos porque en los casos relacionados con la investigación propia ocultaba el compromiso del autor (Bolívar, Cruz y Lopez 2018). En los talleres se analizó exhaustivamente el artículo de Hernández Islas (2015) porque resultó ser un caso en el que se encontraron diferentes usos del se, tanto impersonal como de compromiso personal.

Al mismo tiempo, había que hacer mención al uso del Yo "explícito" (mencionado) e implícito en, por ejemplo, formas verbales en plural (pensamos, creemos) o en el pronombre nosotros y otros como nos. Este es un tema aparentemente de selección gramatical, pero tiene 
un papel fundamental en la construcción discursiva de artículos científicos para fortalecer la agencialidad, la voz propia, y para diferenciarse de otros. El problema se hace mucho más claro al estudiar científicamente el discurso académico porque, cuando examinamos gran variedad de géneros y textos, nos damos cuenta de que el manejo de los pronombres personales como el yo (explícito e implícito) varía principalmente de acuerdo con el grado de experiencia de cada investigador, según su posicionamiento teórico en una comunidad científica, y de los géneros en los que escribe (ver Bolívar en prensa).

\section{El estilo científico y el estilo personal}

Otra de las creencias que había que enfrentar a menudo era que algunos profesores deseaban defender su estilo personal como parte de su posicionamento como ser social. No obstante, la buena intención para que el texto luzca "bonito" (Bolívar y Beke 2011), puede redundar a veces en oraciones extremadamente largas, en fallas en el uso del vocabulário especializado y en vaguedad, porque se introduce lenguaje informal (o coloquial) en un texto que debería ser más directo y formal. Aunque es importante diferenciar entre la retórica de la ciencia y otros tipos de retórica (ver Sánchez Upegui 2011) y estudiar lingüísticamante el lenguaje de la ciencia como se hace, por ejemplo, en la perspectiva sistémico-funcional, (Halliday 1994, 2004), en los talleres se mostró que el estilo científico en educación se puede entender mucho mejor a través del análisis cuidadoso de los artículos publicados dentro del espacio que abarca esta comunidad científica. Esta comunidad nos muestra que los estilos personales existen materializados en el uso diferente de los recursos linguísticos, pero tomando como referencia parámetros generales comunes en la disciplina. Aunque puede parecer elemental, la solución a muchos de los problemas reside en el buen manejo de la puntuación y de los patrones textuales en los que la evaluación tiene un papel central (Bolívar 2001, 2006). La puntuación es otro modo de significación en el que se materializa semióticamente la interacción con el lector porque permite controlar la cantidad de información y las evaluaciones que se hacen a medida que se construye el texto.

\section{Discusión}

Como hemos planteado en este artículo, entrenar a profesores a escribir un buen artículo científico en educación es un processo complejo que trasciende la escritura porque se trata, simultáneamente, de un proceso de construirse como autores y de tomar conciencia de lo que 
significa investigar en una comunidad científica y discursiva a la cual se desea tener acceso. Igualmente, se trata de un proceso en el que cada palabra escrita tiene un valor para indicar un contenido y una relación con otros, con los pares (Beke 2011, Bolívar 2004, 2005ª).

Cuando los pares y los interesados en un problema de investigación leen un artículo traen expectativas basadas en acuerdos internos construídos culturalmente con base en supuestos sobre como debe ser la comunicación científica. Una de estas expectativas en educación es que el artículo científico debe seguir una estructura característica de la investigación empírica (IMRC o IMRDyC), pero en la práctica la fórmula parece extenderse a otros tipos de textos que no son producto de una investigación empírica culminada. Pensamos que aquí hay un tema importante para la discusión porque, después de examinar textos publicados en las revistas de educación, encontramos que el deseo de aplicar esta estructura a otros textos relacionados con el processo de la investigación podría llevar a artículos mal organizados o confusos, con excepciones interesantes.

Nos preguntamos ¿hasta qué punto están claros los investigadores sobre la diferencia entre artículo de investigación y ensayos de investigación? La secuencia IMRC fue fijada por comunidades científicas para asegurarse de que los métodos y resultados estén claros, pero no aplica siempre a textos cuyo objetivo es, por ejemplo, revisar críticamente la literatura o evaluar políticas educativas, en los que se necessita argumentar de otra manera, aunque deberá darse evidencia. En términos teóricos esto implica investigar más a fondo el discurso de los variados tipos de artículos de investigación científica en educación en proyectos sobre el discurso académico y profesional similares a los llevados a cabo en Chile con distintas disciplinas (Parodi 2008; Parodi y Burdiles 2015). También significa comparar las prácticas discursivas y los métodos en la investigación educativa con las de otras disciplinas para ampliar la perspectiva crítica desde la inter y multidisciplinariedad (ver Adoumieh 2014).

Al mismo tiempo, en relación con la competenia lingüística y discursiva de los profesores universitarios, podemos preguntarnos ¿que tipo de entrenamiento formal están recibiendo los investigadores para usar el discurso argumentativo y los recursos linguísticos que les permiten fortalecer su voz propia? Los talleres trajeron a luz una serie de supuestos de los profesores sobre lo que significa la escritura cientítica, especialmente sobre el posicionamento y la impersonalidad, lo cual es un aspecto que también se abre a la discusión porque, por un lado, hay que estudiar las creencias de los profesores sobre su rol de investigadores y los rasgos 
que deben tener los textos $\mathrm{y}$, por otro, contrastar esas creencias con lo que en realidad se encuentra cuando se analizan rigorosamente los textos publicados en las revistas de calidad o las más consultadas. De hecho, un tema que valdría la pena continuar estudiando es el de los recursos linguísticos que traen a la superfície las distintas formas de impersonalidad, especialmente la descripción de los usos del se que es uno de los problemas todavía no resueltos en las gramáticas del español (Montes Giraldo 2003) y que, aunque recomendado como “impersonal”, oculta también la voz del investigador (Bolívar, Cruz y López 2019).

\section{Conclusiones}

Una de las conclusiones más importantes después de estudiar sugerencias sobre como escribir un buen artículo en educación y de llevar a cabo talleres con profesores universitarios y de Escuelas Normales en México, a quienes se les exige publicar "artículos científicos", es que esta tarea no es posible si no hay una investigación empírica terminada, lo cual es el primer punto en las sugerencias para escribir artículos de calidad en educación (Murillo et al. 2017). Pero, al mismo tiempo, hay otra conclusión fundamental relacionada con el entrenamiento de los profesores universitarios en el discurso académico, y tiene que ver con el hecho de que tanto los profesores/investigadores como los instructores deben estar preparados para enfrentarse a la amplia variedad de textos que se construyen en el proceso de la investigación. Esto es clave porque cuando hacemos búsquedas temáticas por internet nos vamos a encontrar con todo tipo de artículos, no siempre de investigaciones empíricas terminadas (que sirven como modelos), y los investigadores deben saber que tienen distintas opciones para publicar (ver Sánchez Upegui 2011). Esta es una realidad, especialmente en el caso de estudiantes de doctorado a quienes se les exige publicar artículos mientras están cursando los estudios. También sucede en Grupos Académicos que presentan avances y se involucran en discusiones teóricas y metodológicas.

Lo anterior puede tener importantes implicaciones. Una de ellas se relaciona con la evaluación de los artículos por los pares. Existe evidencia empírica de que los arbitrajes en educación son bastante severos y que exigen rigurosidad científica mediante el uso de la negación (no tiene, no muestra, no aclara, etc.) (Bolívar 2011), pero no sabemos hasta qué punto las instrucciones de las revistas incluyen orientaciones precisas sobre otros textos. Aparentemente no, porque en la práctica se observa que los profesores tratan de ajustarse a la estructura de artículo científico en casos de ensayos científicos, que podrían tener secuencias argumentativas bien definidas y darían a los investigadores mayor libertad y confianza para 
escribir. Tal vez, el mayor problema que habría que abordar es el de las formas en que los educadores representan lo que significa escribir en la universidad (Bigi, García Romero y Chacón 2018; Martins 2018) e incluso en la escuela, en Educación Básica, donde se construyen inicialmente esas representaciones (Silva-Peña, Tapia e Ibáñez 2016).

Otra implicación es que la escritura de un buen artículo científico en educación requiere el trabajo en equipo de investigadores en educación, la experticia de lingüistas y/o analista del discurso que sean investigadores del discurso académico y científico, y el apoyo de las autoridades cuyo papel es promover la investigación. Una buena parte del trabajo en manuales sobre cómo escribir artículos científicos apunta a explicar el lenguaje científico, a menudo con base en investigaciones y son muy bienvenidos. Pero existe un vacío en educación en cuanto a cómo aproximarse a la gran variedad de textos que no son AC sino ensayos científicos. Pienso que este vacío se puede llenar en gran parte con el trabajo interdisciplinar y esto puede lograrse en talleres especializados en el entrenamiento de buenos escritores y de buenos investigadores al mismo tiempo. Así como en los talleres me he familiarizado, por ejemplo, con lo que significa innovar en educación en México, evaluar aprendizajes, estudiar al plagio, evaluar críticamente políticas educativas en distintos ámbitos, los profesores han aprendido a tomar conciencia crítica de su lenguaje, del valor de un punto aparte y de una coma, de lo que significa posicionarse para tener voz propia y adquirir confianza y, sobre todo, de la importancia de ser cuidadosos porque cada palabra y cada signo de puntuación tiene un valor para otro que lee y juzga la calidad de la investigación y de lo escrito.

\section{Referencias}

Adoumieh, N. (2014). Escribir desde las disciplinas: una experiencia pedagógica en Ciencias Sociales. Paradigma, 35(1), 183-213.

Beke, R. (2008). El discurso académico: la atribución del conocimiento en la investigación educativa. Núcleo, 20 (25), 13-35.

Beke, R. (2011). Las voces de los otros en el discurso académico. Caracas: Universidad Central de Venezuela.

Beke, R. y Bolívar, A. (2009). Certainty and commitment in the construction of academic knowledge in the humanities. En E. Suomela-Salmi y F. Dervin (Eds.), Cross linguistic and cross cultural perspectives on Academic Discourse, pp.33-47. Amsterdam. John Benjamins.

Bigi Osorio, E.; García Romero, M.; \& Chacón Guerrero, E. (2018). Una mirada a las concepciones docentes respecto a las tareas de escritura en la universidad. Akademos 20, 1 y $2,163-185$. 
Bolívar, A. \& Bolet, F. (2011). La introducción y la conclusión em el artículo de investigación. En A. Bolívar y R. Beke (2011). Lectura y escritura para la investigación, pp.93-129. Caracas: Universidad Central de Venezuela. 1 ed. $1^{\text {a }}$ re-impresión 2014.

Bolívar, A. (1999). Los resúmenes para eventos científicos en linguística aplicada en América Latina: estructura e interacción. Opción, 29, 61-81.

Bolívar, A. (2001). The negotiation of evaluation in written text. En M. Scott y G. Thompson (Eds.), Patterns of text. In honour of Michael Hoey (pp. 129-158). Amsterdam/Philadelphia: John Benjamins.

Bolívar, A. (2004). Análisis crítico del discurso de los académicos. Revista Signos, 37 (55), 718.

Bolívar, A. (2005a). Discurso e interacción en el texto escrito. Caracas: Universidad Central de Venezuela, $2^{\text {a }}$ ed. $1^{\text {a }}$ ed. 1994.

Bolívar, A. (2005b). Tradiciones discursivas y construcción del conocimiento en las humanidades. Signo y Seña 14: 67-91.

Bolívar, A. (2006). La función de la evaluación en artículos y ensayos humanísticos. En J. Falk, J. Gille y F. Weichmeister Bermúdez (Coords.) Discurso, interacción e identidad, pp. 109136. Estocolmo: Universidad de Estocolmo.

Bolívar, A. (2011). Funciones discursivas de la evaluación negativa en informes de arbitraje de artículos de investigación en educación. Revista Núcleo 28, 59-89.

Bolívar, A. (en prensa). El plagio y la construcción del conocimiento propio y ajeno en los textos académicos. Monográfico sobre Formas de prevenir el plagio. México: Universidad de las Americas Puebla.

Bolívar, A. Beke, R. y Shiro, M. (2010). Las marcas lingüísticas del posicionamiento en las disciplinas: Estructuras, voces y perspectivas discursivas. En G. Parodi (Ed.). Alfabetización académica y profesional en el siglo XXI: leer y escribir en las disciplinas, pp.95-125. Santiago de Chile: Grupo editorial Planeta Chilena S.A.

Bolívar, A. Cruz Martínez, A. y López Franco, S. (2018). La voz autoral explícita, implícita y oculta en Introducciones y Conclusiones de tesis doctorales. Boletín de Lingüística XXX /49- 50/Ene-Dic. 8-28.

Bolívar, A. y G. Parodi (2015). “Academic and professional discourse”. En M. Lacorte (Ed.). The Routledge Handbook of Hispanic Applied Linguistics, pp. 459- 476. New York and London: Routledge.

Bolívar, A. y R. Beke (Eds.) (2011). Lectura y escritura para la investigación. Caracas: Universidad Central de Venezuela. 1 ed. 1ª re-impresión 2014.

Bustamante, S. (2011). La escritura de textos: un problema gramatical, retórico y psicológico. Paradigma, 32 (2), 39-52.

Carlino, P. (2005). Escribir, leer y aprender en la universidad. Una introducción a la alfabetización académica. Buenos Aires: Fondo de Cultura Económica.

Carlino, P. (2006). La escritura en la investigación. Disponible en http://www.udesa.edu.ar/files/EscEdu/DT/DT19-Carlino.pdf 
Castelló, M. (Coord.) (2007). Escribir y comunicarse en contextos científicos y académicos: Conocimientos y estrategias. Barcelona: Graó.

Castro, M. C. y Sánchez, M. (2016). La formación de investigadores en el área de humanidades: Los retos de la construcción de la voz autoral en la escritura de la tesis de doctorado. Signos $49,30-51$.

Castro, M. C.; Hernández, L.A. y Sánchez, M. (2010). El ensayo como género académico. Una aproximación a las prácticas de escritura en la universidad pública mexicana. En Parodi, G. (Ed.). (2010), Alfabetización académica y profesional en el siglo XXI: Leer y escribir desde las disciplinas, pp.49-70. Santiago de Chile: Editorial Planeta chilena.

Cisneros, M. y Olave Arias, G. (2012). Redacción y publicación de artículos científicos. Enfoque discursivo. Bogotá: Ecoe Ediciones.

Cubo de Severino, L. (Coord.). (2005). Los textos de la ciencia. Principales clases de discurso académico-científico. Córdoba: Comunicarte.

Day, R.A. (2005). Cómo escribir y publicar artículos científicos. $3^{\mathrm{a}}$ ed. En español. M. Sáenz (Trad.). Washington: Organización Panamericana de la Salud.

Fernández, M. B. y Johnson, D. (2015). Investigacion-acción en formación de profesores: desarrollo histórico, supuestos epistemológicos y diversidad epistemológica. Perspectivas, 14 (3), 93-105. https://scielo.conycit.cl

Fløttum, K. (2009). Academic voices in the research article. En E. Suomela- Salmi y F. Dervin (Eds.), Cross-Linguistic and Cross Cultural Perspectives on Academic Discourse, pp. 109122. Amsterdam/Philadelphia: John Benjamins.

García Negroni, M.M. (2008). Subjetividad y discurso científico-académico: acerca de algunas manifestaciones en el artículo de investigación en español. Revista Signos 41, 66: 9-31.

Gee, P. J. (2008). Social linguistics and literacies. Ideology in discourses. London and New York: Routledge. (1 ${ }^{\mathrm{a}}$ ed. 1990, red. 1996).

González, F. (2014). Dificultades en la realización de trabajos de investigación: cómo afrontarlas. Práxis educativa, 1 (18), 275-300.

Halliday, M.A.K. (1994). An introduction to functional grammar. London. Edward Arnold. $2^{\mathrm{a}}$ ed.

Halliday, M.A.K. (2004). The language of science. London: Continuum.

Hernández Islas, M. (2016). El plagio académico en la investigación científica. Consideraciones desde la óptica del investigador de alto nivel. Perfiles educativos, 38 (153), 120-135.

Hernández Islas, M., Ponce Rojo, A.; Ortiz Lefort, V. y Vergara Fregoso, M. (2015). Estrategias docentes emergentes para prevenir el plagio académico en alumnos de pregrado. Un estudio fenomenográfico con profesores experimentados. Consensus, 20 (22), 41-62.

Hernández Ramírez, L.A. (2018). Formas de atribución y grados de argumentatibilidad en la postura autoral en las Conclusiones de artículos científicos. Akademos, 20,1-2, 123-147.

Júarez Núñez, J.M.; Comboni Salinas, S. y Garnique Castro, F. (2010). De la educación especial a la educación inclusiva. Dossier: Procesos educativos en América latina: política, mercado y sociedad. Argumentos (Mx), 23, 62, enero-abril. www.scielo.org.mx/scielo 
Martins, I. (2018). Leer y escribir en la Universidad Simón Bolívar. Akademos 20, 1 y 2, 187206.

Montes Giraldo, J. J. (2003). El “se” del español y sus problemas. Estudios Filológicos, 38, 121137.

Mostaceiro, R. (2014). La construcción de la escritura personal a partir del discurso del otro. Lingua Americana, 8(15), 63-79.

Murillo, F. J.; Martínez-Garrido, C. y Belvi, G. (2017). Sugerencias para escribir un buen artículo científico en educación. REICE. Revista Iberoamericana sobre calidad, eficacia y cambios en educación, 15 (3), 5-34. https://doi.org/10.15366/reice2017.15.3.001

Parodi, G. (Ed.) (2008). Géneros académicos y géneros profesionales: Accesos discursivos para saber y hacer. Valparaíso, Chile: Ediciones Universitarias de Valparaíso, Pontifica Universidad Católica de Chile.

Parodi, G. (Ed.) (2010). Alfabetización académica y profesional en el siglo XXI: leer y escribir desde las disciplinas. Santiago de Chile: Editorial Planeta Chilena S.A.

Parodi, G. y Burdiles, G. (2015). Leer y escribir en contextos académicos y profesionales. Géneros, corpus y métodos. Santiago de Chile: Ariel Planeta Chilena S.A.

Scott, M. \& Thompson, G. (Eds.) (2001). Patterns of text. In honour of Michael Hoey. Amsterdam/Philadelphia: John Benjamins Publishing Company.

Silva-Peña, I.; Tapia, R. \& Ibáñez, M. (2016). Concepciones docentes sobre la escritura en Primer año de Educación Básica. Paradigma, 37 (1), 46-20.

Suomela-Salmi, E. y Dervin, F. (Eds.) (2009). Cross-Linguistic and Cross-cultural perspectives on Academic discourse. Amsterdam/Philapdelphia: John Benjamins Publishing Company.

Swales, J. (1986). Citation analysis and discourse analysis. Applied Linguistics, 7, 39-56.

Swales, J. (1990). Genre analysis: English in academic and research settings. Cambridge: Cambridge University Press.

Titscher, S., Meyer, M., Wodak, R. y Vetter, E. (2000). Methods of text and discourse analysis. London: Sage Publications.

Torres Cañizalez, P.C.; Cobo, B. y Kendry, J. (2017). Tecnología educativa y su papel en el logro de los fines de la educación. EDUCERE, 21 (68), 31-40. https://www.redalyc.org/articulo.oa?id=

UNESCO. (1983). Guía para la redacción de artículos científico destinados a la publicación. Paris: UNESCO. 